\title{
A WEB-BASED MOBILE MEDICAL MONITORING SYSTEM
}

\author{
${ }^{1}$ Department of Electronic and Electrical Engineering, \\ ${ }^{2}$ Department of Medical Physics \\ University College London \\ jp@ee.ucl.ac.uk, http://www.ee.ucl.ac.uk/ jp/ \\ mefry@medphys.ucl.ac.uk, http://www.medphys.ucl.ac.uk/ mefry/
}

J.K. Pollard' ${ }^{1}$, S. Rohman ${ }^{1}$ and M.E. Fry ${ }^{2}$

\begin{abstract}
A hospital-wide mobile medical monitoring system is described. Telemetry devices are attached to patients to acquire, store and process continuous data about their state of health.

Medical staff may examine real-time graphical information and make comparisons with historical data. Parameters may be set to cause an automatic alert to portable devices held by appropriate staff if a patient requires urgent observation.

The system uses the services provided by Internet and Intranet to allow remote supervision and consultation.

A hardware/software prototype has been constructed to demonstrate real-time data acquisition, wireless transmission/reception and connection to the World-Wide Web.

The real-time, supervisory and remote-teaching aspects of the software system are being designed and are in the process of implementation.
\end{abstract}

Keywords: mobile, medical, Web, monitor, SCADA

\section{INTRODUCTION}

An automatic medical monitoring system with decentralised supervisory control and centralised data storage allows focussed attention on patients needs. A data acquisition system of mobile transducers using transceiver communication has a minimum intrusive effect on patients because they can move freely.

This system is to augment the care of patients by continuous monitoring, where required. Medical staff may increase their efficiency in care provision by setting parameters to cause an alert should a patient's condition deteriorate unexpectedly.

Previously recorded data allows comparisons and health trend conclusions to be drawn and it may assist in diagnosis and prognosis.

Remote consultations and out-patient supervision are possible.

If historical data is kept anonymous, it may also be used for remote teaching of medical students [1].

\section{SYSTEM MODEL}

The system model is shown in Figure 1.

Multiple transducers on a single patient monitor different health conditions, such as heart condition, blood pressure, temperature and respiration. The data are processed in a micro-controller with a memory buffer. The data are transmitted in bursts by means of a transceiver, which is carried by the patient.

The data from multiple patients are received and processed by micro-controllers that are connected to the parallel-ports of Personal Computers (PCs). Data buffering in local memory at the receiver is required to cater for different rates of communication in the system.

All computers are connected by a Local Area Network and communicate with each other and a central database server using both Intranet and Internet protocols for World-Wide Web connection [2].

The PCs act as decentralised supervisory controllers and have applications and local storage for the acquired data.

In addition to the automatic monitoring functions, doctors and nurses may enter information about patient monitoring parameters. They can also consult with each other about drugs, diet and other instructions that may be stored on the local computers.

These computers are connected to transceivers that transmit to Personal Digital Assistants (PDAs), pagers and mobile telephones [3]. These mobile wireless devices can be used to remotely monitor patients' condition.

It is also possible to provide automatic notification of unusual changes in monitored data. If certain parameter trigger levels are breached, then 


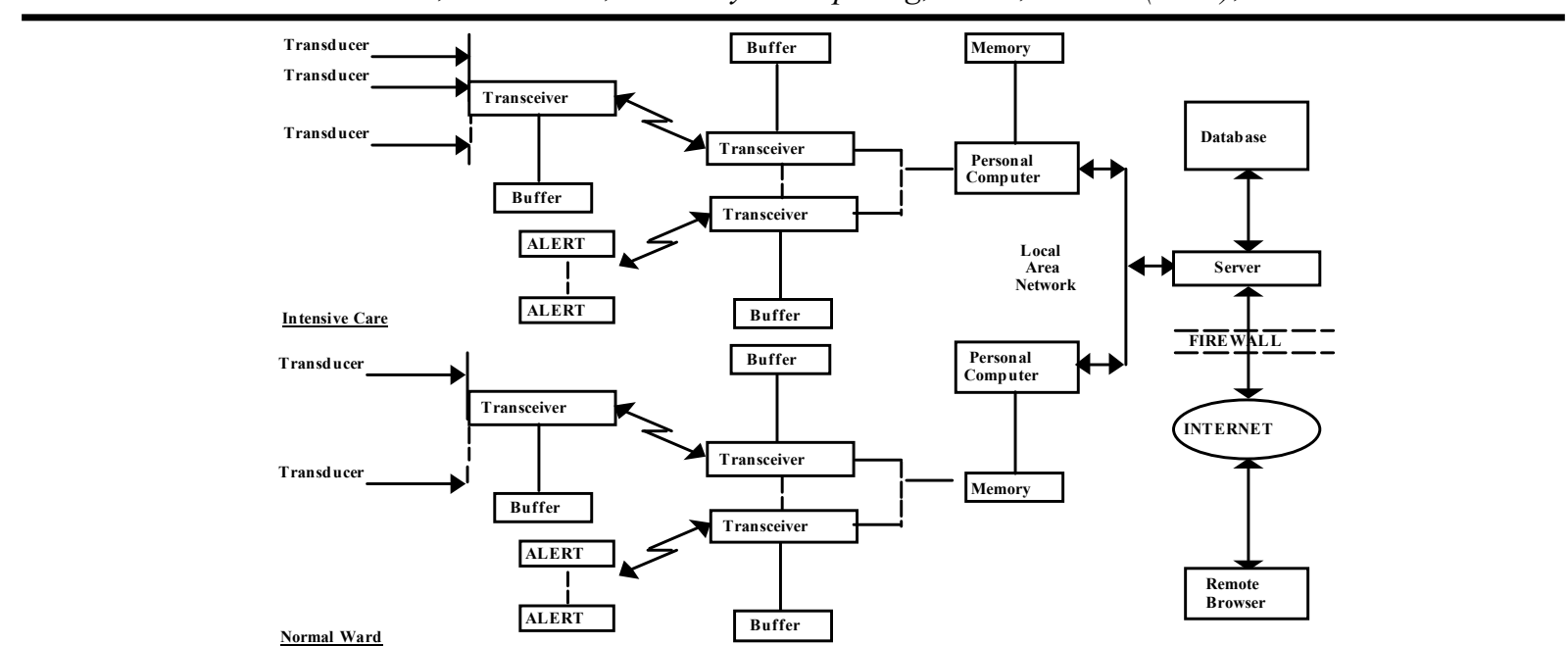

Fig. 1 Mobile medical monitoring system

alert messages may be initiated from the PCs and displayed to appropriate medical staff.

\section{SOFTWARE MODEL}

The medical monitoring system is designed as an augmentation for efficient care of patients. It is not safety critical but, nevertheless, it is required to be robust, resilient, reliable and flexible for enhancement.

Security and confidentiality of patient data is important and so data is compartmentalised to be available only to those who are authorised recipients.

The software on the PC and portable devices allows authorised users (doctors and nurses) to view and edit multiple windows that illustrate current data as monitor graphs, longer term medical charts, patient care plans, current medication and a critical-limits file for alerts. The topology of the software security system is shown in Figure 2.

The security software system allows users to enter name/password into a Web browser and the Web server authenticates this using a password file. Users may be administrators, consultants, doctors, nurses and students. These different types of user may be allowed to access certain "realms" of data. An individual may be restricted to access only certain files. The user is delivered a customised Web page and is supplied with tokens (permits) for navigation through realms of allowed information. Certain users are given permission for parameter entry to allow supervisory control.
Records may be abstracted from PCs to a server computer that communicates with a central database. In addition to the controlled access of localised information, the access to these records is only available to administrators, doctors and nurses.

The medical system is an Intranet that sits behind a "Firewall". Only certain remote clients on the Internet, such as consultants and students, are trusted to access particular data. Permissions from patients are needed.

The real-time software system allows doctors and nurses to enhance patient supervision using a variety of transducers to monitor patient health. The block diagram of the system is shown in Figure 3.

The type of transducer and the parameters of use need to be chosen with the aid of Graphical User Interfaces that are displayed on Web browsers. These are dynamically created by using Webservlets and customised for the monitor type and person. Access to parameter value selection is more

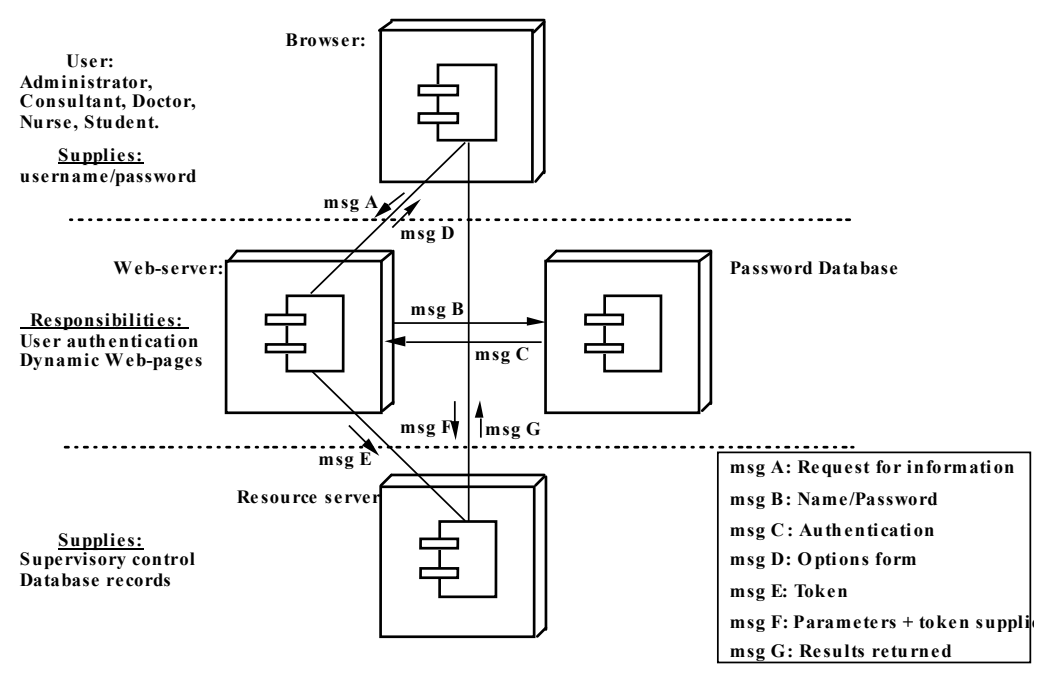

Fig. 2 Security software system 


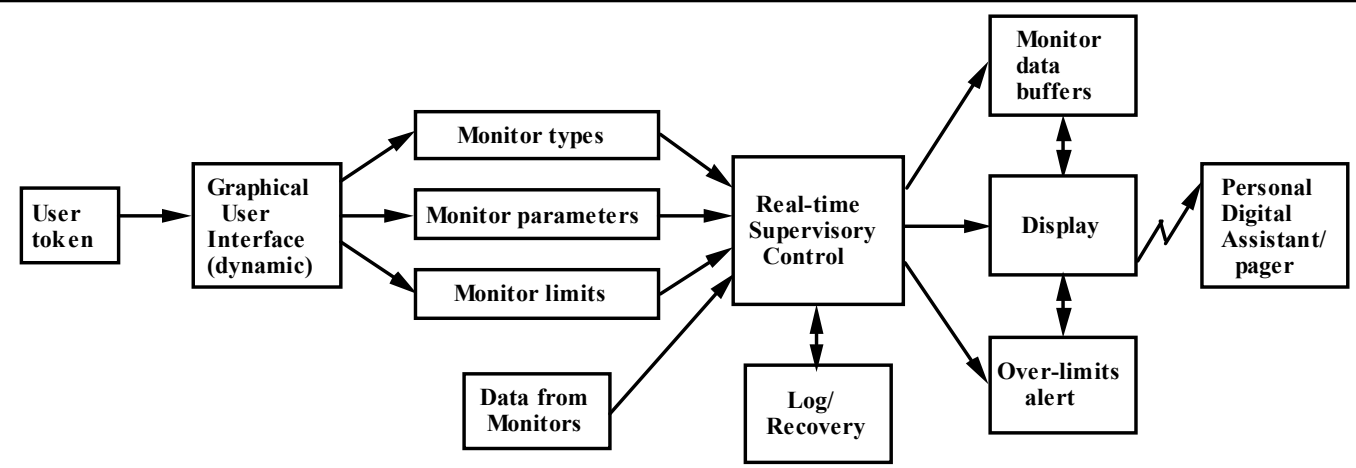

Fig. 3 Real-time software system

tightly controlled than reading of data. Limits on the expected values of monitor data enable health warnings to be triggered should they be exceeded.

The data from the monitors is continuously acquired and stored in temporary buffers. The realtime software acts as a supervisory control that continuously interacts with the data in these buffers. It formats the buffered data so that it can be displayed on request or when triggered by the system. Servlet software is required to format the data so it can be viewed in a Web-browser or with reduced quality if the requesting device is a "thinclient", such as a mobile telephone, pager or a PDA.

The buffered data is passed periodically to a centralised database where records are analysed and stored by a DataBase Management System.

Real-time systems are required to be resilient in the presence of errors. Transactions are logged and a "watch-dog" confirms that the system is working correctly. Data exceptions that may be caused by patient actions in disturbing correct monitor operation need to be distinguished from technical communication errors. The software attempts to recover from these inadvertent data excursions. System failures may require us ers to be informed about the reduction in quality of the information that is available.

The function of the centralised records software system is to record, modify, analyse and retrieve patient information. At the centre is a database. The software system is shown in Figure 4.

This database has two sources of input: human dataentry of information and requests via (telephone) keyboard and mouse, and continuous updates from the real-time software temporary buffers, which store the data that monitor patient health.

A dynamic Web-browser interface is supplied by a Web servlet. This use of the
Intranet allows controlled access and a Graphical User Interface that is widely compatible with a range of display devices. Data entry into the database and modification of details of staff and patients is possible. These entries may record the hospital location and contact information, etc. Patient records and statements of prescribed treatment, evaluation of current health, progress and prognostication describe medical facts that impinge on patient health.

The data that is derived from real-time measurements may require analysis that has the prescribed treatment as a parameter. The results of analysis allow the trends in the data to be observed. Trends that have been monitored over periods of days may produce evidence of success of such treatments.

Snapshot data of monitored output can be used as clear evidence of patient health and its evolution. Such illustrations can be useful for remote medical consultants and student medical practitioners.

Patient records enable health development to be tracked over long time periods and also aid diagnosis when a new problem arises for an individual person.

Anonymous medical records are an important resource for medical research.

Administration summary reports usually require records of patient data to be available.

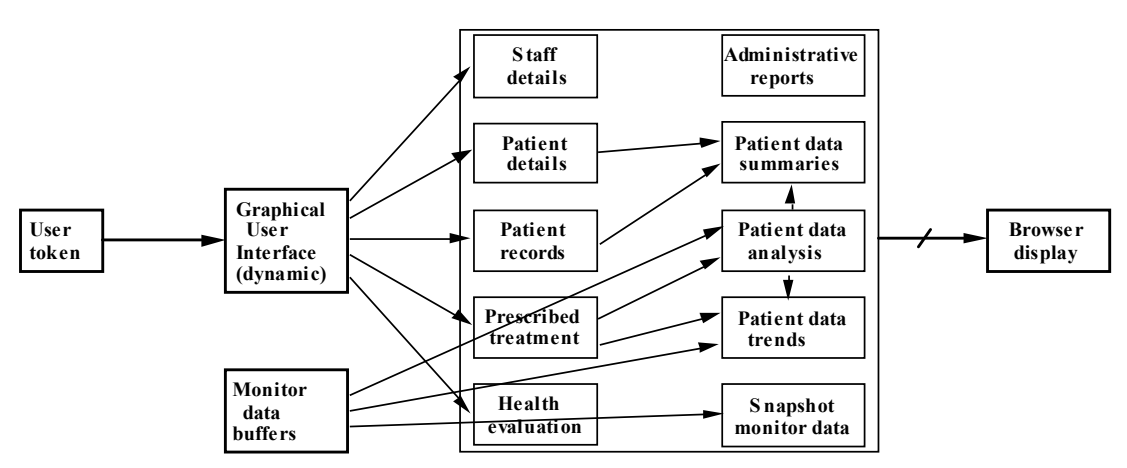

Fig. 4 Records software system 


\section{ACQUISITION HARDWARE}

The generic transceiver circuit, which acquires data from a medical transducer and transmits it, is shown as a block diagram in the upper section of Figure 5. The lower section of this figure is a block diagram of the receiver circuitry.

The transmitter instrumentation pre-amplifier is mounted adjacent to the transducer. The signal DC element is filtered out of the signal, which is
Java program reads the data into memory and the hard disk.

\section{CONCLUSION}

Recent technological advances such as the Internet, Intranet and World-Wide Web have made software available that is widely compatible with heterogeneous computing devices, including thinclients such as PDAs, pagers and mobile telephones. Recent developments in ad-hoc, indoor Wireless Personal Area Networks have made it possible to create economic, mobile monitoring systems. One such real-time Data Acquisition and Supervisory Control system for the medical field is being designed and constructed in hardware and software.

passed to the main amplifier and anti-aliasing filter. A micro-controller containing a 10-bit Analogue-to-Digital Converter (ADC) is programmed to provide an Extended Hamming code $(15,10)$ that provides Forward Error Correction (FEC) of all single errors plus double error detection. A parallel-to-serial converter is used and transmission frames are constructed before being transmitted using Frequency Modulation.

The receiver also has a micro-controller that is programmed to synchronise on the start data pattern and then detect and decode the incoming data. It is placed in the parallel format expected by the PC parallel port.

The interface software on the PC is written in "C" and this acts as a Java Native Interface. A

\section{REFERENCES}

[1] J.K. Pollard, "DATE: A Distribution Aid for a Teaching Environment", Proc. UEF-IEEE Conf.: Electrical \& Electronics Eng. Educ., Davos, Switzerland, (Sept., 2000), pp 4/1-5.

[2] B. Coutinho, M.E. Fry and J.K. Pollard, "Web-controlled instrumentation for educational applications", Proc. IEE Conf. Eng. Educ.: Innovations in Teaching, Learning and Assessment, London, (Jan., 2001).

[3] F. Allegretti, R. Kanani, M.Dixon and V.V. Helenius, "Distributed Applications for Mobile Platforms", M.Eng Thesis, Dept. Electronic and Electrical Engineering, UCL, 2001.

Dr. John Pollard is in the Department of Electronics and Electrical Engineering at University College London, UK. His background is in the design of Integrated Circuits, communication systems and software systems.

In recent years, he has been interested in the use of the World-Wide Web as an enabling technology for remote medical monitoring and for teaching. An integrated combination of hardware and software is necessary to connect a distributed system of mobile computers, Personal Digital Assistants and mobile tele phones with databases and real, physical apparatus. 